\title{
Role played by the SP4 gene in schizophrenia and major depressive disorder in the Han chinese population
}

Jianhua Chen, Kuanjun He, Qingzhong Wang, Zhiqiang Li, Jiawei Shen, Tao Li, Meng Wang, Zujia Wen, Wenjin Li, Yu Qiang, Ti Wang, Jue Ji, Na Wu, Zhiqiao Wang, Bo Zhang, Guoyin Feng, Lin He, Yifeng Xu and Yongyong Shi

\section{Background}

Psychiatric disorders such as schizophrenia and major depressive disorder (MDD) are likely to be caused by multiple susceptibility genes, each with small effects in increasing the risk of illness. Identifying DNA variants associated with schizophrenia and MDD is a crucial step in understanding the pathophysiology of these disorders.

\section{Aims}

To investigate whether the SP4 gene plays a significant role in schizophrenia or MDD in the Han Chinese population.

\section{Method}

We focused on nine single nucleotide polymorphisms (SNPS) harbouring the SP4 gene and carried out case-control studies in 1235 patients with schizophrenia, 1045 patients with MDD and 1235 healthy controls recruited from the Han Chinese population.

\section{Results}

We found that rs40245 was significantly associated with schizophrenia in both allele and genotype distributions $\left(P_{\text {allele }}=0.0005, P_{\text {allele }}=0.004\right.$ after Bonferroni correction; $P_{\text {genotype }}=0.0023, P_{\text {genotype }}=0.0184$ after Bonferroni correction). The rs6461563 SNP was significantly associated with schizophrenia in the allele distributions $\left(P_{\text {allele }}=0.0033\right.$, $P_{\text {allele }}=0.0264$ after Bonferroni correction).

\section{Conclusions}

Our results suggest that common risk factors in the SP4 gene are associated with schizophrenia, although not with $M D D$, in the Han Chinese population.

\section{Declaration of interest}

None.

\section{Copyright and usage}

(c) The Royal College of Psychiatrists 2016.
Schizophrenia and major depressive disorder (MDD) are severe mental disorders with relatively high morbidity and heritability. Schizophrenia affects about $1 \%$ of the world population and has approximately $60-85 \%$ individual heritability. ${ }^{1}$ MDD affects $8-12 \%$ of the population in most countries and has an individual heritability of $40-50 \% .^{2}$ The aetiology of these two psychiatric disorders is still not clear, although genetic factors appear to be possible risk factors. ${ }^{1}$ Several genetic studies have been carried out to search for the possible risk genes of these disorders. Identifying DNA variants associated with schizophrenia and MDD is a crucial step in understanding the pathophysiology of these disorders.

Transcription factor Sp4 (SP4) is a member of the SP1 family of transcription factors, which is highly expressed in the brain and could reasonably play an important role by controlling the transcription of various genes. ${ }^{3,4}$ Previously, SP4 single nucleotide polymorphisms (SNPs) have been found to be associated with schizophrenia, MDD and bipolar disorder in the White population. ${ }^{5-7}$ Rare copy number variations and deletions in the SP4 gene have also been found to be associated with schizophrenia. ${ }^{8,9}$ Furthermore, SP4 has been found to play an important role in the development of cerebellar granule neurons by promoting activity-dependent pruning of dendritic processes. ${ }^{4}$ Recently, using genome-wide association studies (GWAS) and a meta-analysis, Shyn et $a l^{6}$ reported that SP4 was a leading susceptibility candidate gene for MDD, and that it might also be the shared risk factor for both schizophrenia and bipolar disorder. ${ }^{10,11}$ Fuste et al ${ }^{12}$ showed that protein levels of SP1 and SP4 were significantly reduced in first-episode psychosis in lymphocytes, suggesting that these transcription factors were potential peripheral biomarkers of psychotic spectrum disorders in the early stages of the disorder.
Zhou et al $^{13}$ showed that reduced Sp4 expression in mice induced behavioural defects associated with psychiatric disorders. Hypomorphic Sp4 mutant mice displayed robust deficits in both sensorimotor gating and contextual memory. This revealed a novel Sp4 pathway that is essential for hippocampal integrity and modulates behavioural processes.

We hypothesised that the SP4 gene might contribute to the pathogenesis of schizophrenia or MDD by modulating sensorimotor gating. We carried out our association study in the Han Chinese population. We comprehensively genotyped nine SNPs - rs1018954, rs10233357, rs2073534, rs2107448, rs2285941, rs3735440, rs40245, rs10255890 and rs6461563 - among 1235 patients with schizophrenia, 1045 patients with MDD and 1235 normal controls in the Han Chinese population.

\section{Method}

\section{Patients}

The sample set included 1235 unrelated patients with schizophrenia (805 men and 430 women), 1045 unrelated patients with MDD (729 men and 316 women) and 1235 healthy controls (665 men and 570 women). All these samples were recruited from the Han Chinese population.

Patients were recruited from the Shanghai Mental Health Center. The mean age of patients with schizophrenia was 36.4 years (s.d. $=9.0)$, and age at disease onset was 20.4 years (s.d.=8.7); the mean age of patients with MDD was 34.4 years $($ s.d. $=12.1)$, and age at disease onset was 30.0 years (s.d. $=6.9$ ); the mean age of the healthy controls was 30.6 years $($ s.d. $=11.4$ ) (Table 1). Two independent psychiatrists from the Shanghai Mental Health Center interviewed each patient. Diagnoses were 


\begin{tabular}{|c|c|c|c|c|c|c|c|}
\hline & \multicolumn{3}{|c|}{$n$} & \multicolumn{2}{|c|}{ Age, years } & \multicolumn{2}{|c|}{ Age at disease onset, years } \\
\hline & Men & Women & Total & Mean & s.d. & Mean & s.d. \\
\hline Patients with schizophrenia & 805 & 430 & 1235 & 36.4 & 9.0 & 20.4 & 8.7 \\
\hline Patients with MDD & 729 & 316 & 1045 & 34.4 & 12.1 & 30.0 & 6.9 \\
\hline Healthy controls & 665 & 570 & 1235 & 30.6 & 11.4 & N/A & N/A \\
\hline
\end{tabular}

made strictly according to the DSM-IV criteria based on the Structured Clinical Interview for DSM-IV Axis I Disorders (SCID-I) ${ }^{14}$ Patients with the following exclusion criteria were not recruited: history of substance use; neurological illness; mental retardation; mood disorder; psychotic disorder due to general medical condition. Healthy controls were randomly selected from the general Han Chinese population and were recruited from the local community residents by means of announcements on bulletin boards. Before collecting blood samples, the healthy controls were also interviewed by two independent psychiatrists using the SCID-I to exclude any lifetime psychiatric disorders. The healthy controls were excluded only if they had a severe medical illness. Written informed consent was obtained from all participants. The study proposal and protocol were reviewed and approved by the local Ethical Committee of Human Genetics.

\section{Selection and genotyping of SNPS}

The SP4 gene is located on chromosome 7p15; it spans about $86.79 \mathrm{~kb}$ of DNA and consists of six exons and five introns. It contains several SNPs as identified by the International SNP Consortium (www.ncbi.nlm.nih.gov/SNP), the Ensembl genome browser (www.ensembl.org) and the UCSC Genome Browser (http:// genome.ucsc.edu/cgi-bin/hgGateway). We selected the common tag SNPs from the HapMap (http:// hapmap.ncbi.nlm.nih.gov/) Han Chinese population in Beijing. ${ }^{15}$ The relationship between tag SNPs and the SP4 gene is shown in online Fig. DS1.

In accordance with the study protocol, the QuickGene DNA whole blood kit L (FujiFilm) was used to extract genomic DNA from peripheral blood samples. Tag SNP selection was performed with the Haploview software (Broad Institute; www.broadinstitute. org), with $r^{2} \geqslant 0.5$ and minor allele frequency $\geqslant 0.05$. $^{16,17}$ Nine SNPs (rs1018954, rs10233357, rs2073534, rs2107448, rs2285941, rs3735440, rs40245, rs10255890 and rs6461563) were selected according to previous reports and the results of tag SNPs selection. ${ }^{7,16}$ Coverage of the whole gene by those tag SNPs was $72 \%$, as analysed by the Tagger software (www. broadinstitute.org/mpg/tagger/server.html). The location of the nine SNPs in the studied region is shown in online Table DS1. All SNPs were genotyped with the TaqMan SNP Genotyping Assays and the Fluidigm EP1 platform. All probes and primers were designed using the Assay by Design or Assay on Demand service of Life Technologies. SNPs were determined by the genotype calls of each sample with a call rate $>95 \%$.

\section{Statistical analysis}

Association analysis with regard to the SNPs was carried out with the SHEsis software (http://analysis.bio-X.cn ${ }^{18,19}$ ), a user-friendly platform equipped with a suite of highly efficient analytical tools designed for association studies that can analyse linkage disequilibrium, haplotype construction and genetic association at polymorphism loci. SHEsis was integrated with the partitionligation-combination-subdivision expectation maximisation algorithm, designed for efficient estimation of haplotypes constructed from large numbers of biallelic or multi-allelic loci in diploid individuals. All tests were two-tailed, with statistical significance set at $P<0.05$. $P$-values were adjusted using the Bonferroni correction method.

\section{Population stratification analysis}

We used the STRUCTURE software, version 2.3.4 (http:// pritchardlab.stanford.edu/structure.html ${ }^{20-22}$ ) to perform the population stratification analysis. Additionally, we genotyped the data of 79 randomly selected SNPs for the stratification analysis. We obtained the data for these 79 SNPs from 522 HapMap samples, including 174 samples from Utah residents with ancestry from Northern and Western Europe in the United States (CEU), 209 samples from Yoruba, in Ibadan, Nigeria (YRI) and 139 samples from the Han Chinese population in Beijing (CHB) (International HapMap Project; http://hapmap.ncbi.nlm.nih.gov/cgi-perl/ gbrowse/hapmap28_B36/). ${ }^{15}$ We used the STRUCTURE software to isolate the distinct populations using the genotype data of these 79 SNPs from the 522 HapMap samples. We applied the admixture and correlated frequencies models, with a burn-in length of 10000 and Markov chain Monte Carlo repeats of 10000 .

\section{Results}

\section{Linkage disequilibrium}

The pairwise linkage disequilibrium among the nine investigated SNPs was different in the different sample sets. SNPs with $\mathrm{D}^{\prime}>0.95$ in both disorder sample sets were categorised in the same block. No specific haplotype block was identified, as shown in Fig. 1. (See also online Figs DS3 and DS4.)

\section{Single site association analysis}

The rs2107448 SNP was excluded from further analysis because it was not in Hardy-Weinberg equilibrium in the healthy controls, the significance threshold being $P<0.05$.

Table 2 shows the results for the remaining eight polymorphic SNPs: rs40245 was significantly associated with schizophrenia in both allele and genotype distributions $\left(P_{\text {allele }}=0.0005, P_{\text {allele }}=\right.$ 0.004 after Bonferroni correction; $P_{\text {genotype }}=0.0023, P_{\text {genotype }}=$ 0.0184 after Bonferroni correction); rs6461563 was significantly associated with schizophrenia in the allele distributions $\left(P_{\text {allele }}=\right.$ $0.0033, P_{\text {allele }}=0.0264$ after Bonferroni correction; $P_{\text {genotype }}=$ $0.0078, P_{\text {genotype }}=0.0624$ after Bonferroni correction); rs3735440 showed allelic or genotypic significance with both schizophrenia and MDD before Bonferroni correction, and rs10233357 showed allelic significance with MDD before Bonferroni correction, but these association were eliminated after Bonferroni correction.

Separate analyses for men and women are shown in online Tables DS2 and DS3. In the male samples, rs40245 was significantly associated with schizophrenia in both allele and genotype distributions, and rs6461563 was significantly associated with schizophrenia in the allele distributions. 


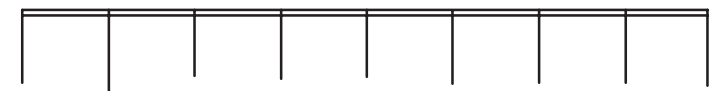

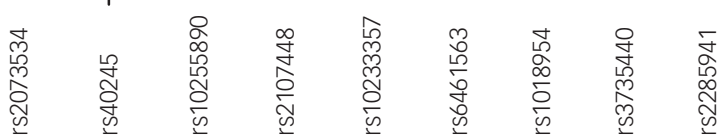

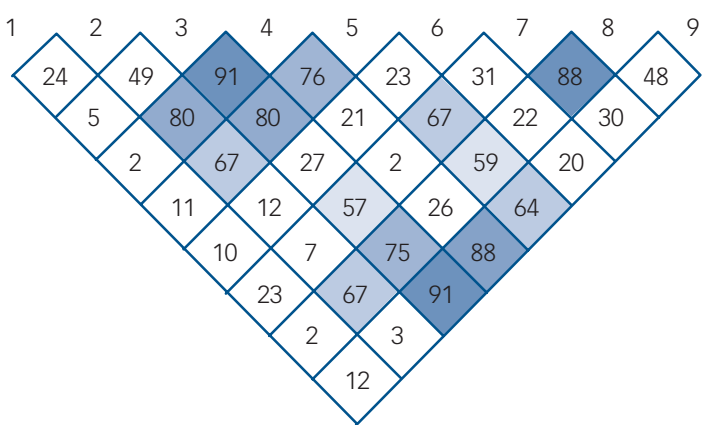

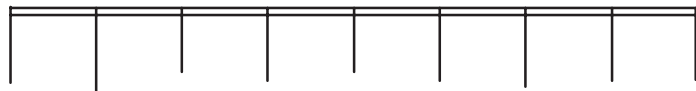

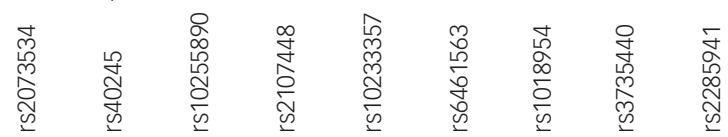

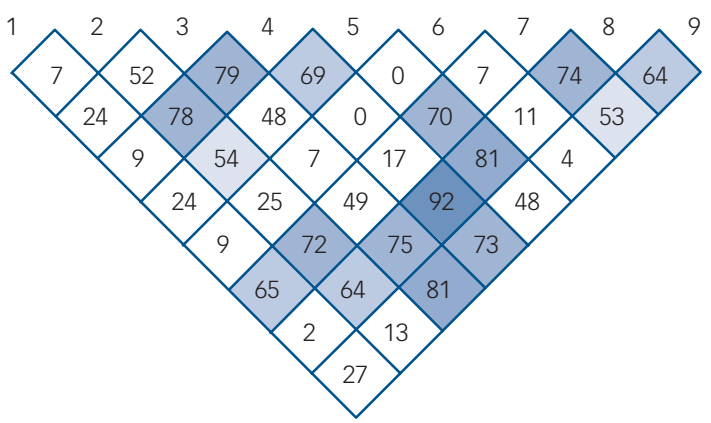

Fig. 1 Linkage disequilibrium plot of the nine single nucleotide polymorphisms in the samples associated with (a) schizophrenia and (b) major depressive disorder.

\begin{tabular}{|c|c|c|c|c|c|c|c|c|c|c|}
\hline SNP & Allele frequency & \multirow[t]{2}{*}{ OR } & \multirow[t]{2}{*}{$95 \% \mathrm{Cl}$} & \multirow[t]{2}{*}{$P_{\text {allele }}$} & \multirow[t]{2}{*}{$\begin{array}{c}\text { Corrected } \\
P^{\mathrm{a}}\end{array}$} & \multicolumn{3}{|c|}{ Genotype frequency } & \multirow[t]{2}{*}{$P_{\text {genotype }}$} & \multirow[t]{5}{*}{$\begin{array}{c}\text { Corrected } \\
P^{a}\end{array}$} \\
\hline rs2073534 & $\mathrm{C}$ & & & & & CC & $\mathrm{CT}$ & $\mathrm{TT}$ & & \\
\hline Schizophrenia & $471(0.193)$ & 1.072 & $0.93-1.24$ & \multirow{3}{*}{$\begin{array}{l}0.342 \\
0.192\end{array}$} & & $53(0.044)$ & $365(0.300)$ & $800(0.657)$ & \multirow{3}{*}{$\begin{array}{l}0.603 \\
0.415\end{array}$} & \\
\hline MDD & $346(0.168)$ & \multirow{2}{*}{0.902} & \multirow{2}{*}{$0.77-1.05$} & & & $34(0.033)$ & $278(0.270)$ & 719 (0.697) & & \\
\hline Control & $441(0.183)$ & & & & & $50(0.041)$ & $341(0.283)$ & $816(0.676)$ & & \\
\hline rs40245 & A & & & & & AA & AT & $\Pi$ & & \\
\hline Schizophrenia & $324(0.137)$ & 1.364 & $1.14-1.63$ & 0.0005 & 0.004 & $30(0.025)$ & $264(0.223)$ & $888(0.751)$ & 0.0023 & 0.0184 \\
\hline MDD & $224(0.110)$ & 1.061 & $0.88-1.03$ & 0.545 & & $9(0.009)$ & $206(0.202)$ & $804(0.789)$ & 0.414 & \\
\hline Control & $253(0.104)$ & & & & & $15(0.012)$ & $223(0.184)$ & $975(0.804)$ & & \\
\hline rs10255890 & C & & & & & AA & $A C$ & $\mathrm{CC}$ & & \\
\hline Schizophrenia & $643(0.266)$ & 0.958 & $0.84-1.09$ & 0.508 & & $638(0.529)$ & $495(0.410)$ & $74(0.061)$ & 0.371 & \\
\hline MDD & $520(0.256)$ & 0.906 & $0.79-1.04$ & 0.149 & & $553(0.544)$ & $408(0.401)$ & $56(0.055)$ & 0.147 & \\
\hline Control & $670(0.275)$ & & & & & $641(0.526)$ & $486(0.399)$ & $92(0.075)$ & & \\
\hline rs10233357 & C & & & & & $\mathrm{AA}$ & $A C$ & $\mathrm{CC}$ & & \\
\hline Schizophrenia & $925(0.328)$ & 0.918 & $0.82-1.03$ & 0.148 & & $464(0.383)$ & $571(0.471)$ & $177(0.146)$ & 0.280 & \\
\hline MDD & 889 (0.434) & 1.140 & $1.01-1.28$ & 0.0320 & 0.256 & 337 (0.329) & $487(0.475)$ & $201(0.196)$ & 0.108 & \\
\hline Control & $971(0.402)$ & & & & & $441(0.365)$ & $563(0.466)$ & $204(0.169)$ & & \\
\hline rs6461563 & G & & & & & GG & GT & $\pi$ & & \\
\hline Schizophrenia & $771(0.342)$ & 1.204 & $1.06-1.36$ & 0.0033 & 0.0264 & $141(0.125)$ & 489 (0.434) & $497(0.441)$ & 0.0078 & 0.0624 \\
\hline MDD & $578(0.307)$ & 1.025 & $0.90-1.17$ & 0.713 & & $80(0.085)$ & $418(0.444)$ & $444(0.471)$ & 0.718 & \\
\hline Control & $702(0.302)$ & & & & & $103(0.088)$ & $496(0.426)$ & $565(0.485)$ & & \\
\hline rs1018954 & A & & & & & AA & AT & $\pi$ & & \\
\hline Schizophrenia & $192(0.079)$ & 1.169 & $0.94-1.45$ & 0.156 & & $7(0.006)$ & $178(0.147)$ & $1023(0.847)$ & 0.326 & \\
\hline MDD & $162(0.080)$ & 1.176 & $0.94-1.47$ & 0.158 & & $5(0.005)$ & $152(0.150)$ & $857(0.845)$ & 0.358 & \\
\hline Control & $166(0.069)$ & & & & & $4(0.003)$ & $158(0.131)$ & $1045(0.866)$ & & \\
\hline rs3735440 & $G$ & & & & & AA & $A G$ & GG & & \\
\hline Schizophrenia & $224(0.094)$ & 0.784 & $0.65-0.94$ & 0.0101 & 0.0808 & 979 (0.822) & $200(0.168)$ & $12(0.010)$ & 0.0127 & 0.1016 \\
\hline MDD & $186(0.093)$ & 0.775 & $0.64-0.94$ & 0.0104 & 0.0832 & $824(0.824)$ & $166(0.166)$ & $10(0.010)$ & 0.0137 & 0.1096 \\
\hline Control & $281(0.117)$ & & & & & $932(0.775)$ & $259(0.215)$ & $11(0.009)$ & & \\
\hline rs2285941 & G & & & & & GG & GT & TT & & \\
\hline Schizophrenia & $734(0.302)$ & 1.113 & $0.98-1.26$ & 0.090 & & $120(0.099)$ & $494(0.407)$ & $600(0.494)$ & 0.245 & \\
\hline MDD & $537(0.262)$ & 1.125 & $0.80-1.04$ & 0.171 & & $77(0.075)$ & $383(0.374)$ & $565(0.551)$ & 0.401 & \\
\hline Control & $682(0.280)$ & & & & & $106(0.087)$ & $470(0.386)$ & $641(0.527)$ & & \\
\hline
\end{tabular}




\section{Population stratification analysis}

Online Fig. DS2 shows the population stratification analysis when $\mathrm{K}=3$ : each angle represents a possible independent ancestry and the differently coloured dots represent the individuals in the population we want to analyse. The combined populations of CEU, YRI and our samples display a clearly stratified pattern (Fig. DS2(a)), and our samples of the two disorders and healthy controls are evenly distributed in the triangle indicating that there is no obvious significant stratification in the population (Fig. DS2(b)). The results were consistent with one another when $\mathrm{K}$ ranged from 2 to 5 . We could therefore conclude that our positive results were unlikely to be caused by population stratification.

\section{Discussion}

Psychiatric disorders are likely to be caused by multiple susceptibility genes, each with small effects in increasing the risk of illness. ${ }^{23}$ In this study, we discovered an association of SP4 variants with schizophrenia. Our main finding was a significant association between the SP4 gene and schizophrenia in the Han Chinese population. We confirmed that two SNPs, rs40245 $\left(P_{\text {allele }}=0.0005\right.$, odds ratio $(\mathrm{OR})=1.364,95 \%$ confidence interval (CI) $1.14-1.63)$ and $\mathrm{rs} 6461563\left(P_{\text {allele }}=0.0033, \mathrm{OR}=1.204,95 \%\right.$ CI 1.06-1.36), were associated with schizophrenia in a casecontrol study with large sample sizes of a Han Chinese origin. We also found that rs3735440 showed allelic and genotypic significance with both schizophrenia and MDD before Bonferroni correction, whereas rs10233357 showed allelic significance with MDD before Bonferroni correction. However, the association was too weak and they were eliminated after Bonferroni correction. We found that rs40245 and rs6461563 were more sensitive in men with schizophrenia than in women. However, the OR was in the same direction between the two genders. The female sample size was 430 , which might not be large enough to detect the differences. Sample size might be one of the main reasons for the negative results. However, possible gender differences could not be excluded, and a larger female sample size is needed to reach sufficient statistical power.

SP4, located on chromosome 7p15 (start: 21,467,652, end: $21,554,440)$, encodes the brain-specific, zinc finger transcription factor Sp4. Sp4 recognises GC-rich sequences in the promoters of a variety of genes, ${ }^{24}$ where a susceptibility locus was suggested for a broad spectrum of human mental disorders. Zhou et $a l^{7}$ genotyped 506 patients with bipolar disorder and 507 controls, and established that four SNPs in SP4 (rs40245, rs12673091, rs1018954 and rs3735440) displayed significant association with bipolar disorder. We recruited more case and control samples in our study, and found that SNP rs40245 was a susceptibility locus for schizophrenia. At the same time, we found that rs3735440 was significantly associated with schizophrenia and MDD before Bonferroni correction, and rs10233357 was associated with MDD before Bonferroni correction. Evidence from clinical, epidemiological and molecular genetic studies has suggested that some genetic risk factors are shared between neuropsychiatric disorders. Our study also suggested that there might be overlap of genetic factors in major psychiatric disorders. Common variants nominally showed cross-disorder effects for the adultonset disorders (schizophrenia and MDD). These studies provided direct evidence for SP4 as a susceptibility gene for mental disorders, which implied that SP4 might have some important function in the biological processes of neuronal development in humans. SP1 and SP4 transcription factors regulate gene expression in many biological processes including neuronal development and function. ${ }^{25,26}$ In contrast to the ubiquitous expression pattern of the $S P 1$ gene, $S P 4$ is broadly expressed in neurons of the central nervous system and it shows higher expression in the cerebellum and hippocampus. ${ }^{13,27-29}$ In patients with schizophrenia, it was reported that SP1 mRNA levels altered in lymphocytes and some areas of the brain. ${ }^{30}$

In addition, SP4 expression was reduced in the prefrontal cortex and cerebellum of patients with bipolar disorder, providing evidence that this gene might be relevant in psychiatric diseases with psychotic features. ${ }^{31}$ Recently, Fuste $e t$ al ${ }^{12}$ assessed protein and gene expression levels of SP1 and SP4 in peripheral blood mononuclear cells of patients with first-episode psychosis and in healthy controls. They found that SP1 and SP4 levels were significantly reduced in patients compared with healthy controls, suggesting that these transcription factors might be potential peripheral biomarkers of psychotic spectrum disorders in the early stages. Shyn et al ${ }^{6}$ carried out a meta-analysis of three Europeanancestry MDD GWAS data-sets: the Sequenced Treatment Alternatives to Relieve Depression $\left(\mathrm{STAR}^{\star} \mathrm{D}\right)$ collaborative study, the Genetics of Recurrent Early-Onset Depression (GenRED) project and the publicly available Genetic Association Information Network-MDD data-set. An intronic SNP (rs17144465) in SP4 was observed for association in the meta-analysis. In our study two SNPs, rs3735440 and rs10233357, were found to be associated with MDD before Bonferroni correction, which might imply a nominal association with MDD. However, there was no association after Bonferroni correction.

So far, many functional studies have been conducted regarding the expression of SP4. The Sp4 protein is degraded with the activation of glutamate receptors. ${ }^{32}$ Reduced expression of the SP4 gene in mice exhibited decreased granule cell density in the dentate gyrus of the hippocampus, ${ }^{3}$ deficits in sensorimotor gating and contextual learning, ${ }^{13}$ suggesting a possible behavioural deficit, ${ }^{27}$ and association with schizophrenia and other psychiatric disorders. ${ }^{13,33-37}$ SP4 may also play a role in glutamate-induced neurotoxicity. ${ }^{32,38}$ An imbalance in dopamine and glutamate pathways may induce the emergence of these psychiatric disorders. All this evidence strongly suggests a key role for SP4 in mental disorders. Although the two risk polymorphisms rs40245 and rs6461563 are both located in the intron region, there may be some association with other functional variants. SNPs in non-coding areas may also affect gene splicing, transcription factor binding and mRNA degradation.

Our results suggest an association between SP4 and schizophrenia, although not between SP4 and MDD, in the Han Chinese population. We have shown that the same genetic factors can be shared by different mental disorders. Although our sample size was relatively large and we analysed a considerable sample size and achieved association significance of common variants, we lack further evidence from functional experiments to support our findings and to show the role of SP4 or related pathways in the pathogenesis of mental disorders.

\section{Funding}

This work was supported by the National Natural Science Foundation of China (31325014, $81130022,81171271,81272302,31000553,81121001,31240001,31271140)$, the National 863 project (2012AA02A515, 2012AA021802), the National Basic Research Program (973 Program, 2015CB559100), the Program for Changjiang Scholars and Innovative Research Team in University (IRT1025), the Foundation for the Author of National Excellent Doctoral Dissertation of China (201026), the Shanghai Rising-Star Program (12QA1401900), the Shanghai Municipal Natural Science Foundation (11ZR1431300), the Shanghai Key Laboratory of Psychotic Disorders (13dz2260500), the project of the Shanghai Mental Health Center (2012-YJ-08), the Youth Research Project of Shanghai Health and Family Planning Commission (20134Y082), Shu Guang project supported by Shanghai Municipal Education Commission (20134Y082), Shu Guang project supported by Shanghai Municipal Education Jiao Tong University Liberal Arts and Sciences Cross-Disciplinary Project (13JCRZO2). 


\section{Acknowledgements}

We are deeply grateful to all who took part in this study, and to the psychiatrists for their help in recruiting patients and diagnosing schizophrenia and MDD in our patient cohort.

Jianhua Chen, MD, PhD, Shanghai Key Laboratory of Psychotic Disorders, Shanghai Mental Health Center, Shanghai Jiao Tong University School of Medicine, and Bio-X Institutes, Key Laboratory for the Genetics of Developmental and Neuropsychiatric Disorders (Ministry of Education), Shanghai Jiao Tong University, Shanghai, China; Kuanjun He, PhD, Qingzhong Wang, PhD, Zhiqiang Li, PhD, Jiawei Shen, $P h D$, Tao Li, PhD, Meng Wang, MSC, Zujia Wen, PhD, Wenjin Li, PhD, Yu Qiang, MSC, Ti Wang, PhD, Jue Ji, BS, Na Wu, BS, Zhiqiao Wang, BS, Bo Zhang, BS, Bio-X Institutes, Key Laboratory for the Genetics of Developmental and Neuropsychiatric Disorders (Minis Guoyin Feng, BS, Shanghai Key Laboratory of Psychotic Disorders, Shanghai Menta Health Center, Shanghai Jiao Tong University School of Medicine, Shanghai, China; Lin He, PhD, Bio-X Institutes, Key Laboratory for the Genetics of Developmental and Neuropsychiatric Disorders (Ministry of Education), Shanghai Jiao Tong University, Shanghai, China; Yifeng Xu, MD, MSC, Shanghai Key Laboratory of Psychotic Disorders, Shanghai Mental Health Center, Shanghai Jiao Tong University School of Medicine, Shanghai, China; Yongyong Shi, PhD, Shanghai Key Laboratory of Psychotic Disorders, Shanghai Mental Health Center, Shanghai Jiao Tong University School of Medicine Bio-X Institutes, Key Laboratory for the Genetics of Developmenta and Neuropsychiatric Disorders (Ministry of Education), Shanghai Jiao Tong University, Shanghai, Shanghai Changning Mental Health Center, Shanghai, Institute of Neuropsychiatric Science and Systems Biological Medicine, Shanghai Jiao Tong University, Shanghai, and Institute of Social Cognitive and Behavioral Sciences, Shanghai Jiao Tong University, Shanghai, China

Correspondence: Yongyong Shi, Bio-X Institutes, Shanghai Jiao Tong University, Central Little White House, 1954 Huashan Road, Shanghai 200030, China. Email: shiyongyong@gmail.com; Yifeng Xu, Shanghai Mental Health Center, Shanghai Jiao Tong University School of Medicine, 600 Wan Ping Nan Road, Shanghai 200030, China. Email: hyyyyb@gmail.com

First received 31 Aug 2013, final revision 12 Oct 2014, accepted 7 Nov 2014

\section{References}

1 Burmeister M, McInnis MG, Zöllner S. Psychiatric genetics: progress amid controversy. Nat Rev Genet 2008; 9: 527-40.

2 O'Donovan MC, Craddock NJ, Owen MJ. Genetics of psychosis; insights from views across the genome. Hum Genet 2009; 126: 3-12.

3 Zhou X, Qyang Y, Kelsoe JR, Masliah E, Geyer MA. Impaired postnatal development of hippocampal dentate gyrus in Sp4 null mutant mice. Genes Brain Behav 2007; 6: 269-76.

4 Ramos B, Gaudillière B, Bonni A, Gill G. Transcription factor Sp4 regulates dendritic patterning during cerebellar maturation. Proc Natl Acad Sci U S A 2007; 104: 9882-7.

5 Shi J, Potash JB, Knowles JA, Weissman MM, Coryell W, Scheftner WA, et al. Genome-wide association study of recurrent early-onset major depressive disorder. Mol Psychiatry 2011; 16: 193-201.

6 Shyn Sl, Shi J, Kraft JB, Potash JB, Knowles JA, Weissman MM, et al. Nove loci for major depression identified by genome-wide association study of Sequenced Treatment Alternatives to Relieve Depression and meta-analysis of three studies. Mol Psychiatry 2011; 16: 202-15.

7 Zhou X, Tang W, Greenwood TA, Guo S, He L, Geyer MA, et al. Transcription factor SP4 is a susceptibility gene for bipolar disorder. PLOS One 2009; 4: e5196.

8 Tam GW, van de Lagemaat LN, Redon R, Strathdee KE, Croning MD, Malloy $M P$, et al. Confirmed rare copy number variants implicate novel genes in schizophrenia. Biochem Soc Trans 2010; 38: 445-51.

9 Zhou X, Nie Z, Roberts A, Zhang D, Sebat J, Malhotra D, et al. Reduced NMDAR1 expression in the Sp4 hypomorphic mouse may contribute to endophenotypes of human psychiatric disorders. Hum Mol Genet 2010; 19: 3797-805.

10 Blackwood DH, Fordyce A, Walker MT, St Clair DM, Porteous DJ, Muir WJ. Schizophrenia and affective disorders - cosegregation with a translocation at chromosome 1q42 that directly disrupts brain-expressed genes: clinical and P300 findings in a family. Am J Hum Genet 2001; 69: 428-33.

11 Millar JK, Christie S, Anderson S, Lawson D, Hsiao-Wei Loh D, Devon RS, et al. Genomic structure and localisation within a linkage hotspot of Disrupted In Schizophrenia 1, a gene disrupted by a translocation segregating with schizophrenia. Mol Psychiatry 2001; 6: 173-8.

12 Fusté $M$, Pinacho R, Meléndez-Pérez I, Villalmanzo N, Villalta-Gil V, Haro JM et al. Reduced expression of SP1 and SP4 transcription factors in periphera blood mononuclear cells in first-episode psychosis. J Psychiatr Res 2013; 47: 1608-14.

13 Zhou X, Long JM, Geyer MA, Masliah E, Kelsoe JR, Wynshaw-Boris A, et al. Reduced expression of the Sp4 gene in mice causes deficits in sensorimotor gating and memory associated with hippocampal vacuolization. $\mathrm{Mol}$ Psychiatry 2005; 10: 393-406.
14 First MB, Spitzer RL, Gibbon M, Williams JBW. Structured Clinical Interview for DSM-IV-TR Axis I Disorders, Research Version, Patient Edition. (SCID-I/P). Biometrics Research, New York State Psychiatric Institute, 2002.

15 International HapMap Consortium. The International HapMap Project. Nature 2003; 426: 789-96.

16 de Bakker PI, Yelensky R, Pe'er I, Gabriel SB, Daly MJ, Altshuler D. Efficiency and power in genetic association studies. Nat Genet 2005; 37: 1217-23.

17 de Bakker PI, Burtt NP, Graham RR, Guiducci C, Yelensky R, Drake JA, et al. Transferability of tag SNPs in genetic association studies in multiple populations. Nat Genet 2006; 38: 1298-303.

18 Shi YY, He L. SHEsis, a powerful software platform for analyses of linkage disequilibrium, haplotype construction, and genetic association at polymorphism loci. Cell Res 2005; 15: 97-8.

19 Li Z, Zhang Z, He Z, Tang W, Li T, Zeng Z, et al. A partition-ligationcombination-subdivision EM algorithm for haplotype inference with multiallelic markers: update of the SHEsis (http://analysis.bio-x.cn). Cell Res 2009; 19: 519-23.

20 Falush D, Stephens M, Pritchard JK. Inference of population structure using multilocus genotype data: linked loci and correlated allele frequencies. Genetics 2003; 164: 1567-87.

21 Falush D, Stephens M, Pritchard JK. Inference of population structure using multilocus genotype data: dominant markers and null alleles. $\mathrm{Mol}$ Ecol Notes 2007; 7 574-8.

22 Hubisz MJ, Falush D, Stephens M, Pritchard JK. Inferring weak population structure with the assistance of sample group information. $\mathrm{Mol}$ Ecol Resour 2009; 9: 1322-32.

23 Cannon TD, Keller MC. Endophenotypes in the genetic analyses of mental disorders. Annu Rev Clin Psychol 2006; 2: 267-90.

24 Heisler LE, Torti D, Boutros PC, Watson J, Chan C, Winegarden N, et al. CpG Island microarray probe sequences derived from a physical library are representative of $\mathrm{CpG}$ Islands annotated on the human genome. Nucleic Acids Res 2005; 33: 2952-61.

25 Bouwman P, Philipsen S. Regulation of the activity of Sp1-related transcription factors. Mol Cell Endocrinol 2002; 195: 27-38.

26 Black AR, Black JD, Azizkhan-Clifford J. Sp1 and krüppel-like factor family of transcription factors in cell growth regulation and cancer. J Cell Physiol 2001; 188: $143-60$

27 Supp DM, Witte DP, Branford WW, Smith EP, Potter SS. Sp4, a member of the $\mathrm{Sp} 1$-family of zinc finger transcription factors, is required for normal murine growth, viability, and male fertility. Dev Biol 1996; 176: 284-99.

28 Heintz N. Gene expression nervous system atlas (GENSAT). Nat Neurosc 2004; 7: 483

29 Zhu $H$, Nguyen VT, Brown AB, Pourhosseini A, Garcia AV, van Bilsen M, et al. A novel, tissue-restricted zinc finger protein (HF-1b) binds to the cardiac regulatory element (HF-1b/MEF-2) in the rat myosin light-chain 2 gene. $\mathrm{MO}$ Cell Biol 1993; 13: 4432-44.

30 Ben-Shachar D, Karry R. Sp1 expression is disrupted in schizophrenia; a possible mechanism for the abnormal expression of mitochondrial complex I genes, NDUFV1 and NDUFV2. PLOS One 2007; 2: e817.

31 Pinacho R, Villalmanzo N, Roca M, Iniesta R, Monje A, Haro JM, et al. Analysis of $\mathrm{Sp}$ transcription factors in the postmortem brain of chronic schizophrenia: a pilot study of relationship to negative symptoms. J Psychiatr Res 2013; 47: 926-34

32 Mao X, Yang SH, Simpkins JW, Barger SW. Glutamate receptor activation evokes calpain-mediated degradation of Sp3 and Sp4, the prominent Spfamily transcription factors in neurons. J Neurochem 2007; 100: 1300-14.

33 Braff DL, Geyer MA, Swerdlow NR. Human studies of prepulse inhibition of startle: normal subjects, patient groups, and pharmacological studies. Psychopharmacology (Berl) 2001; 156: 234-58.

34 Geyer MA, Krebs-Thomson K, Braff DL, Swerdlow NR. Pharmacological studies of prepulse inhibition models of sensorimotor gating deficits in schizophrenia: a decade in review. Psychopharmacology (Berl) 2001; 156: 117-54.

35 Dempster EL, Toulopoulou T, McDonald C, Bramon E, Walshe M, Wickham H, et al. Episodic memory performance predicted by the $2 \mathrm{bp}$ deletion in exon 6 of the "alpha 7-like" nicotinic receptor subunit gene. Am J Psychiatry 2006; 163: $1832-4$

36 Robles O, Blaxton T, Adami H, Arango C, Thaker G, Gold J. Nonverbal delayed recognition in the relatives of schizophrenia patients with or without schizophrenia spectrum. Biol Psychiatry 2008; 63: 498-504.

37 Daumas $\mathrm{S}$, Halley $\mathrm{H}$, Lassalle JM. Disruption of hippocampal CA3 network: effects on episodic-like memory processing in C57BL/6J mice. Eur J Neurosci 2004; 20: 597-600.

38 Mao X, Moerman-Herzog AM, Wang W, Barger SW. Differential transcriptional control of the superoxide dismutase-2 kappaB element in neurons and astrocytes. J Biol Chem 2006; 281: 35863-72. 